\section{Cutaneous Manifestations of Primary Immunodeficiency Diseases in Libyan Children}

\author{
Safa Suleman Elfaituri ${ }^{1 *}$ and Idris Matoug ${ }^{2}$ \\ ${ }^{1}$ Department of Dermatology, Benghazi University, Benghazi, Libya \\ ${ }^{2}$ Department of Pediatrics, Benghazi University, Benghazi, Libya
}

\begin{abstract}
Primary Immunodeficiency Diseases (PIDs) are serious genetically determined diseases that usually present in early childhood. The skin may be involved in PIDs and cutaneous changes may be the presenting signs and serve as important clues for diagnosis. The objective of this study was to determine the frequency and nature of cutaneous alterations in Libyan children with PIDs. A retrospective study included pediatric patients with PID seen between 2007 and 2014 at immunology department of pediatric hospital in Benghazi, Libya. Data were obtained from the patients files. Sex, age, family history and consanguinity were recorded. Dermatological involvements were grouped according to their etiology.

Sixty patients were studied, $55 \%$ males and $45 \%$ females, of whom $90 \%$ had onset of their disease before the first year of life. PIDs were classified as phagocytic $(62 \%)$; chronic granulomatous disease, neutropenia and leukocyte adhesion defect. Well-defined syndromes with immunodeficiency $(21 \%)$ included Hyper-lgE syndrome, Ataxia telangiectasia, Wiskott-Aldrich syndrome and DiGeorge anomaly. Agammaglobulinemia, common variable immune deficiency and Transient hypogammaglobulinemia of infancy constituted humoral defect $(12 \%)$. Diseases of immune dysregulation included Chediak-Higashi syndrome (3\%).

Defects in innate immunity as Epidermodysplasia verruciformis is $2 \%$. Majority $(87 \%)$ had developed skin disease which was the presenting problem in $47 \%$. Cutaneous infections were the most prevalent manifestations $(80 \%)$ followed by eczematous dermatitis $(12 \%)$, granuloma (8\%) erythroderma (3\%) and autoimmune diseases $(2 \%)$. In conclusion, knowledge of PIDs cutaneous features may aide in the early detection and management of these serious, life-threatening diseases.
\end{abstract}

Keywords: Benghazi; Children; Libya; Primary immunodeficiency; Skin

\section{Introduction}

Primary Immunodeficiency Diseases (PIDs) comprise a number of serious and rare diseases that result from variable genetic defects in

*Corresponding author: Safa Suleman Elfaituri, Department of Dermatology, Benghazi University, Benghazi, Libya, Tel: +218 925496566; E-mail: selfaitoury@yahoo.co.uk

Citation: Elfaituri SS, Matoug I (2017) Cutaneous Manifestations of Primary Immunodeficiency Diseases in Libyan Children. J Clin Dermatol Ther 4: 029.

Received: March 21, 2017; Accepted: May 24, 2017; Published: June 08, 2017 immune system characteristically observed in infancy or in children. Early diagnosis of a PID is important because it can lead to lifesaving treatment, prevention of complications, and significant improvement in the quality of life. The skin may be involved in PIDs and the dermatologist may be the first to diagnose an immune defect [1,2]. Only a few studies describing the spectrum of skin disorders in PID are available and no similar studies have been reported from Libya. The objective of this study was to determine the frequency and nature of cutaneous changes in Libyan children with PIDs.

\section{Method and Materials}

This was a retrospective study and included pediatric patients with PIDs seen between 2007 and 2014 at immunology department of pediatric hospital in Benghazi, Libya. Diagnoses of PIDs were made according to the World Health Organization criteria, [3] and in most of the patients were done abroad. Sex, age, age at onset of disease, family history of disease and consanguinity of their parents were obtained from the clinical files of the patients or by interview. Informed consent was obtained from parents to publish images. Response to treatment was not included in this study.

Diagnoses were grouped according to their primary immune defect according to Classification from the International Union of Immunological Societies Expert Committee for Primary Immunodeficiency [4]. Dermatological involvements were grouped according to the etiology of their most prominent sign.

\section{Results}

Sixty patients were studied, 55\% males and $45 \%$ females, of whom $90 \%$ had onset of their disease before the first year of life. Sixty percent of the patients had positive family history of similar PIDs and $58 \%$ of their parents were relatives. PIDs were classified as phagocytic, well-defined syndromes with immunodeficiency, humoral, diseases of immune dysregulation and defects in innate immunity (Figure 1). Phagocytic PIDs (62\%) included Chronic Granulomatous Disease (CGD), neutropenia and Leukocyte Adhesion Defect (LAD). Well-defined syndromes with immunodeficiency (21\%) Included Hyper-Ige Syndromes (HIES), Ataxia Telangiectasia (AT), Wiskott-Aldrich Syndrome (WAS), and DiGeorge anomaly. Agammaglobulinemia (AG), Common Variable Immune Deficiency (CVID) and Transient Hypogammaglobulinemia of infancy (THG) constituted humoral defect $(12 \%)$. Chediak-Higashi Syndrome (CHS) represent diseases of immune dysregulation (3\%) and Epidermodysplasia Verruciformis (EDV) represents the defect in innate immunity (2\%) (Figure 2).

Eighty seven percent had developed skin disease, and the cutaneous alterations were the presenting problem in $47 \%$ (Figure 3). Cutaneous infections were the most prevalent manifestations, seen in $80 \%$ followed by eczematous dermatitis (12\%), granuloma (8\%) erythroderma (3\%) and autoimmune diseases (2\%) (Figure 4).

Among skin infections; bacterial was the commonest, seen in all patients with HIES, $93 \%$ of CGD and 60\% of AG. Skin abscesses predominated in HIES (100\%) and CGD (75\%). Perianal abscesses and fistulas were observed in 30\% of CGD. Variable bacteria were isolated 


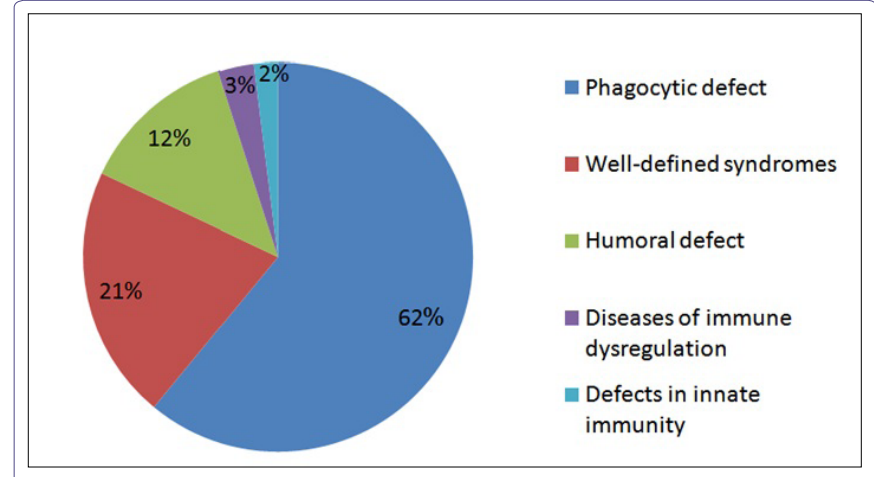

Figure 1: Primary immunodeficiency disorders groups.

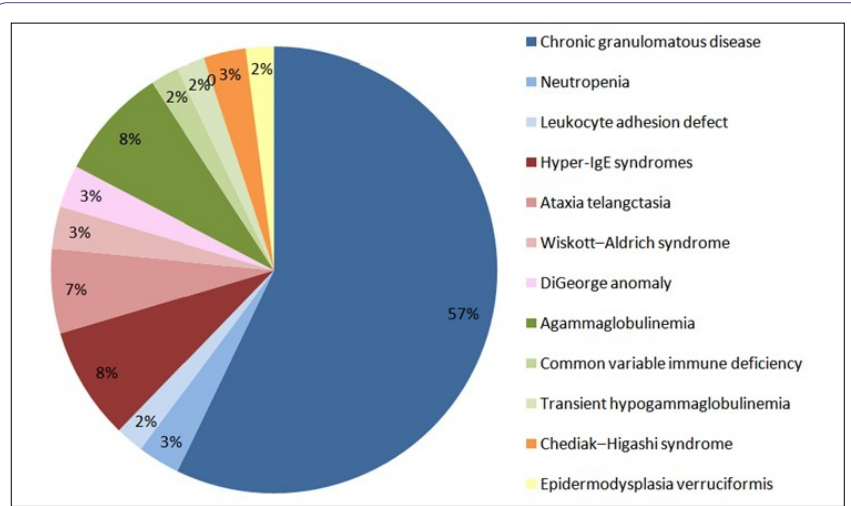

Figure 2: Primary immunodeficiency disorders.

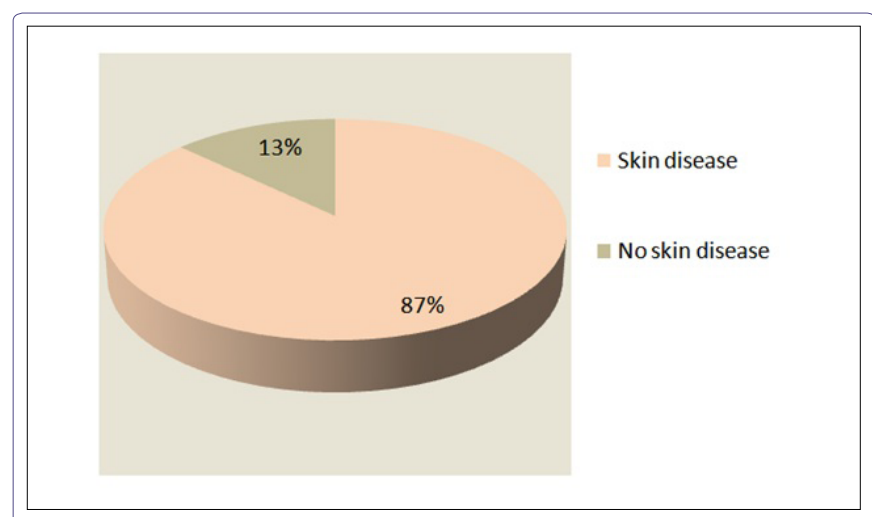

Figure 3: Skin involvement in PIDs.

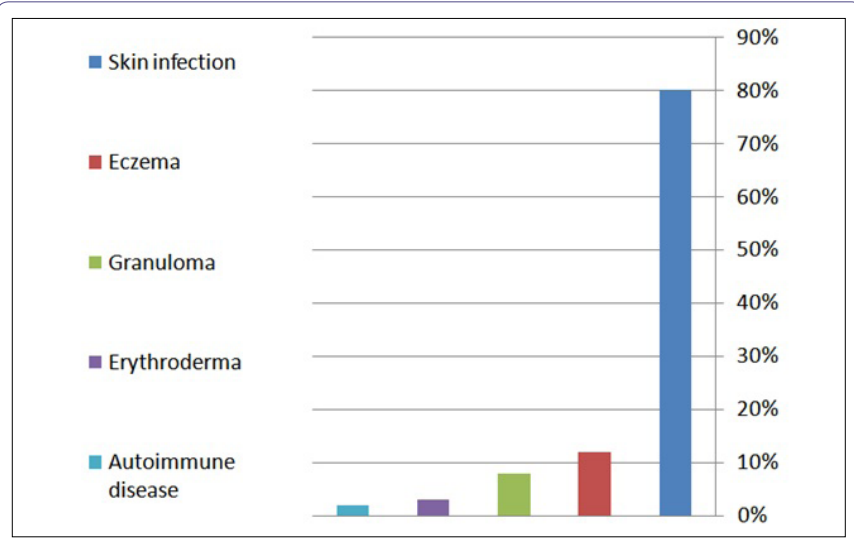

Figure 4: Cutaneous manifestations of PIDs. including Staphylococcus aureus, E. coli, Serratia, Klebsiella, and Pseudomonas but unfortunately bacterial culture was not done in all infections. BCGitis was seen in $20 \%$ and disseminated BCG disease in $15 \%$ of CGD. Fungal infections were mostly due to candidiasis (100\%) of HIES. Extensive tinea corporis was seen in two patients; HIES and CGD. Cutaneous spread of aspergillosis from chest wall was seen in a CGD patient. Two brothers with HIES had suffered from recurrent herpes simplex infection and Kaposi varicelliform eruption. Mucocutaneous leishmaniasis was reported in an AT patient.

Figures 5-14 show variable skin infections in PIDs Eczema was seen in all HIES and WA Spatients. Cutaneous granulomas were observed in 15\% of CGD (Figure 15). Autoimmune diseases including vitiligo and thrombocytopenic purpura were seen in a CVID patient (Figure 16). Two PIDs patients presented with erythroderma; HIES with generalized dermatitis and CGD patient with disseminated BCG disease.

Table 1 demonstrate the cutaneous manifestation in PIDs.

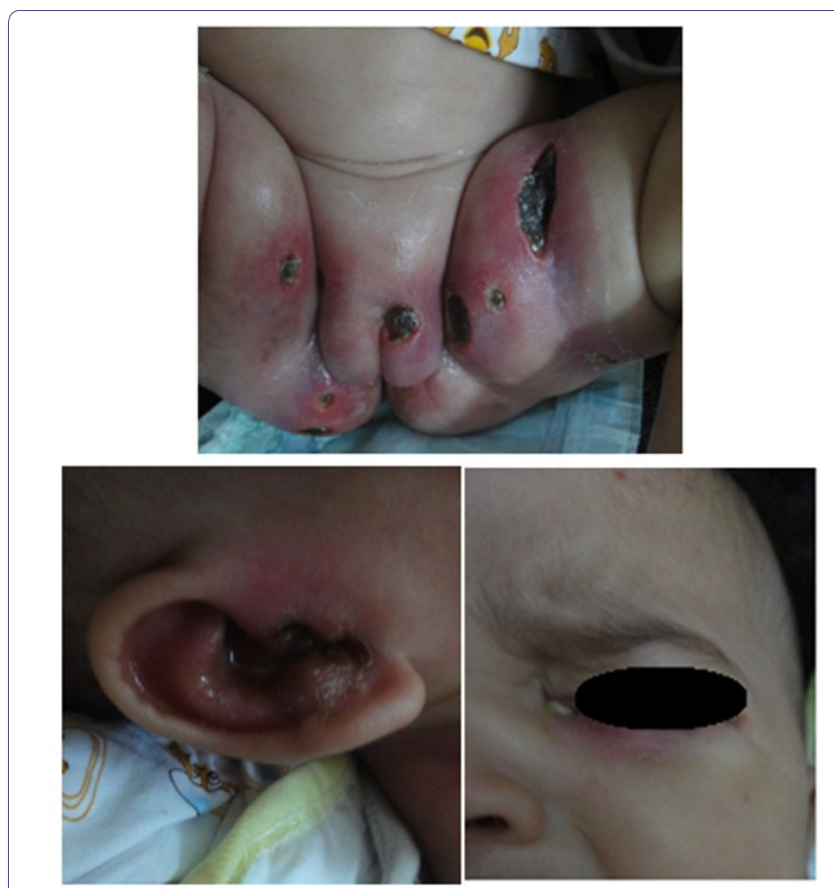

Figure 5: Ecthymagangrenosum with ear and eye infection in a neutropenic infant (pseudomonas was isolated from all sites).

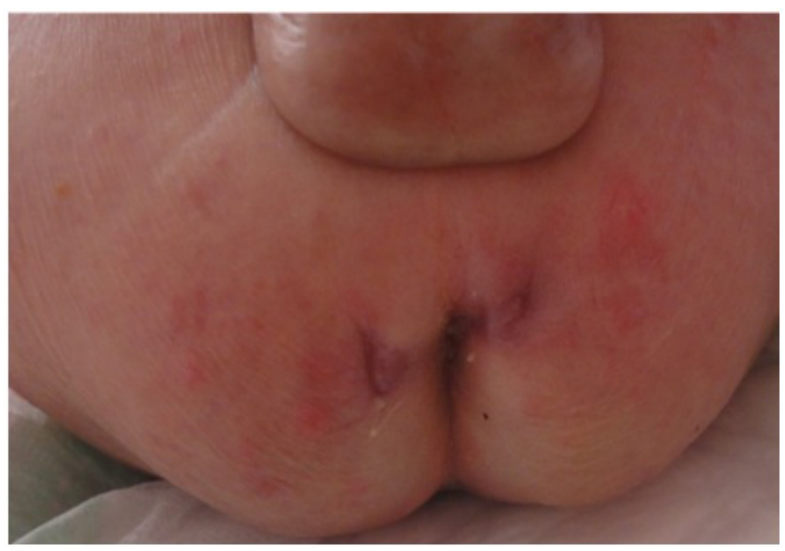

Figure 6: Perianal abscesses and fistulas in CGD. 


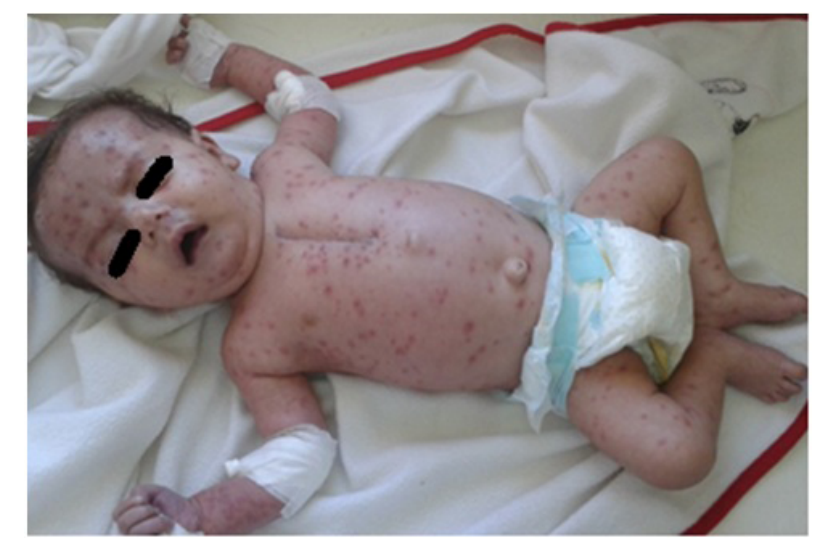

Figure 7: DiGeorge with chickenpox infection.

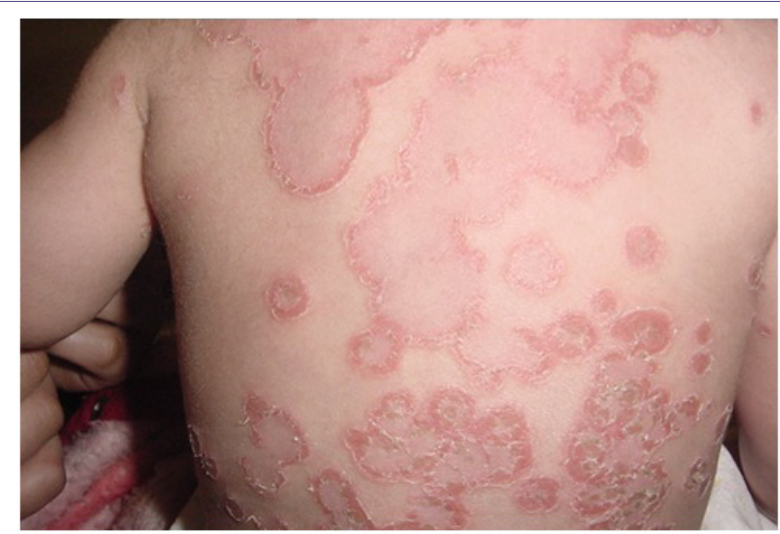

Figure 8: Extensive tinea corporis in CGD.
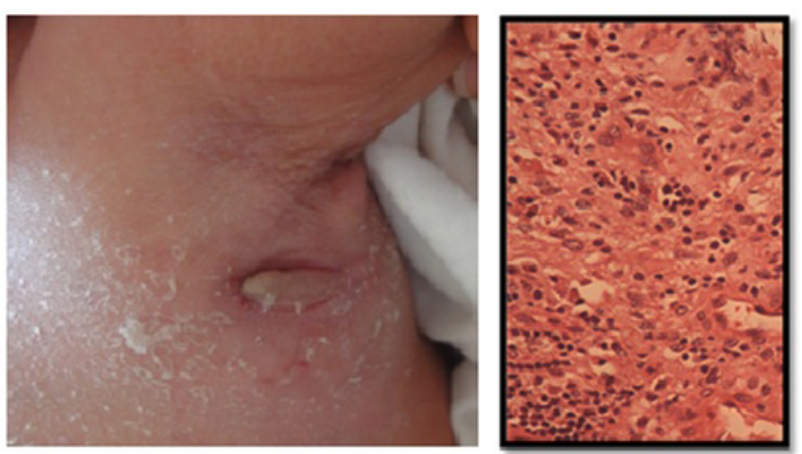

Figure 9: Axillary lymphadenitis with scrofuloderma in CGD.

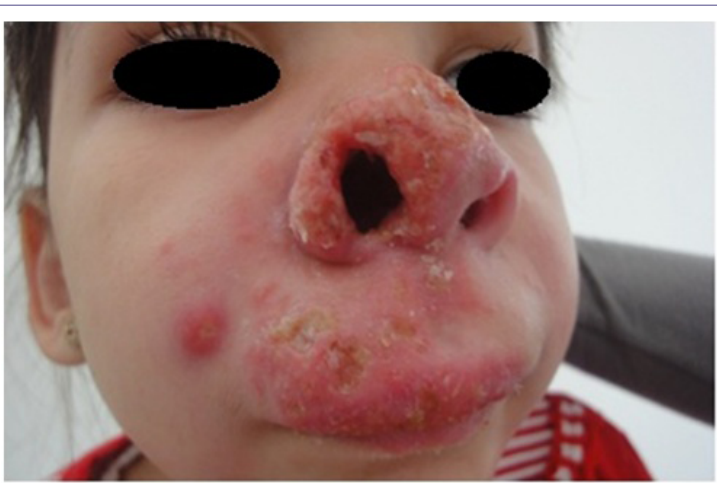

Figure 10: Mucocutaneous leishmania in AT.

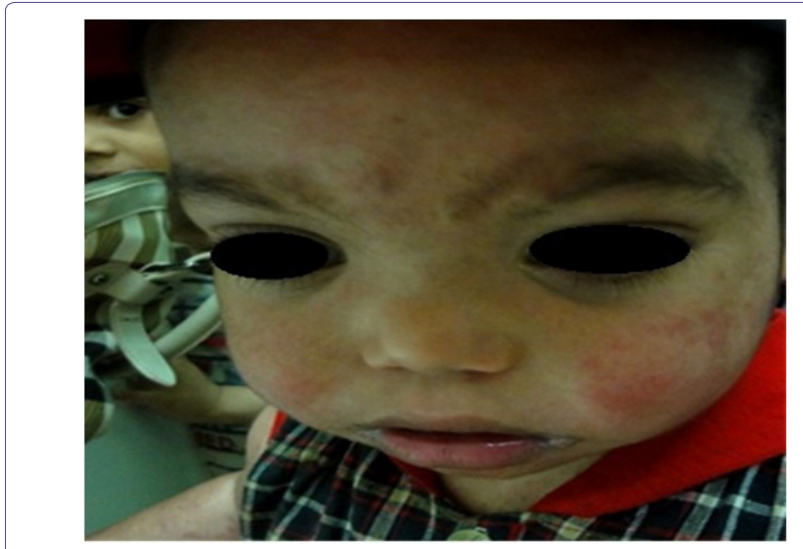

Figure 11: Coarse facial features with eczema and angular cheilitis in HIES

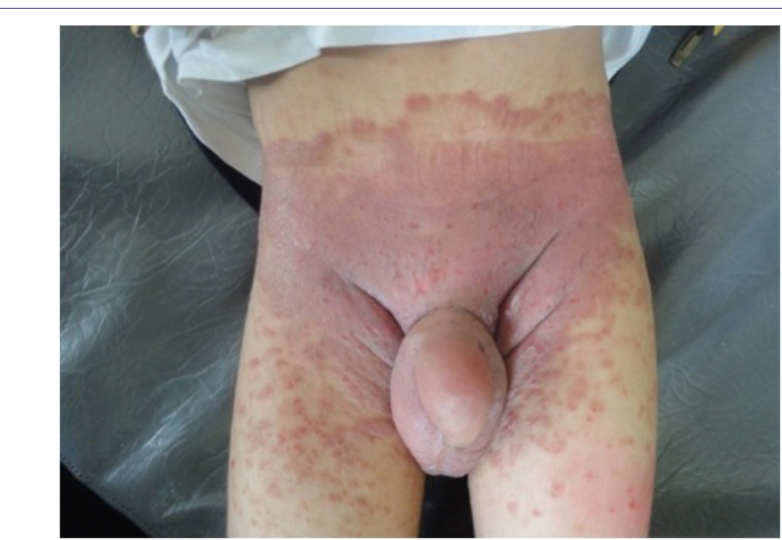

Figure 12: Chronic eczema with tinea corporis in HIES.

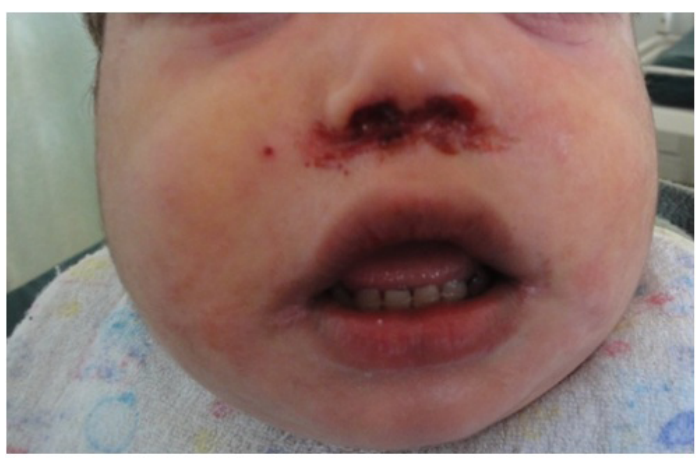

Figure 13: Eczema with impetigo around the nose in HIES

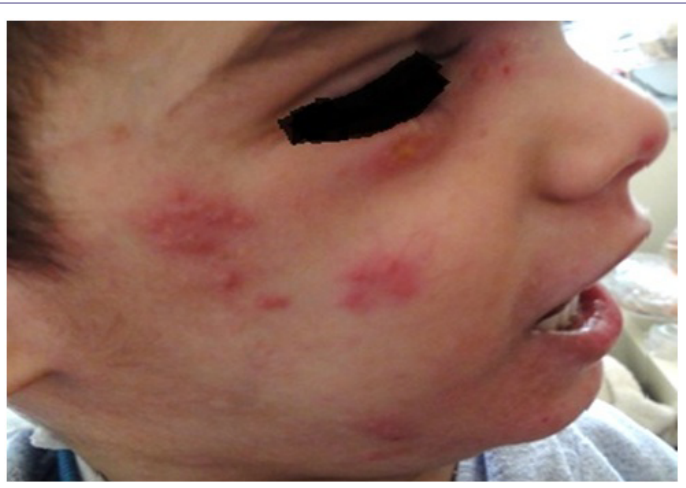

Figure 14: Recurrent herpes simplex infection in HIES 


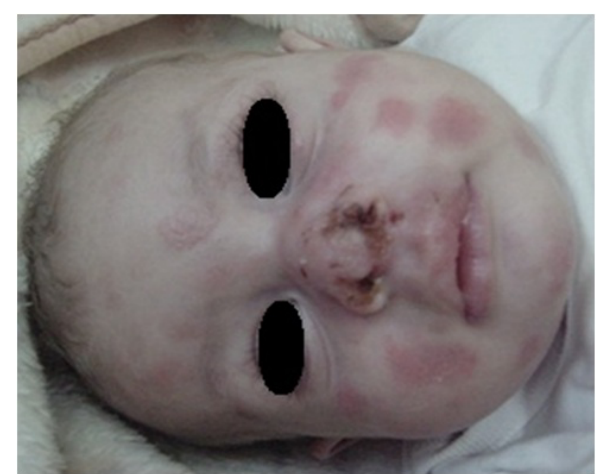

Figure 15: Skin granuloma in CGD with destructive infection of nares.

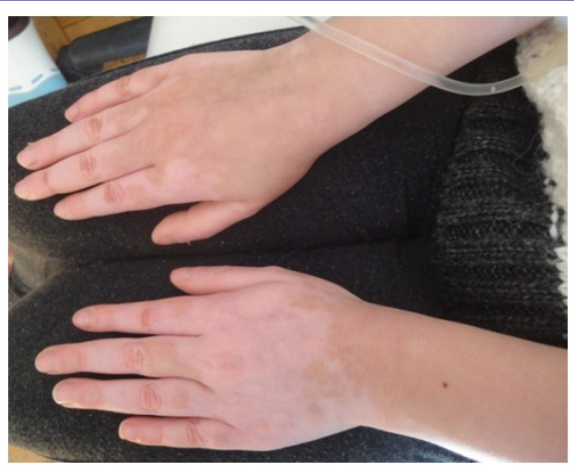

Figure 16: Vitiligo in CVID.

\section{Discussion}

PIDs are uncommon diseases characterized by variable genetic immune defects mostly observed in children. The cutaneous alterations were the presenting problem in $47 \%$ of the patients, higher than what is reported by Hermazewski and Webster $(21 \%)$ and by Moin A, et al., (32\%) [5]. Cutaneous finding may be the clue for the diagnosis of PIDs because of their early presentation, high frequency and their easy access for examination [5-7]. Skin infections are one of the most common cutaneous manifestations of PIDs [8]. In our study, cutaneous infection (80\%) was higher than Berron-Ruiz study results [6]. Pyogenic infections of the skin occur frequently in PIDs, in this study, bacterial infections were the main cutaneous infections. Abscesses predominated in HIES and CGD and they tended to be multiple, recurrent and difficult to control. Abscesses in HIES and especially perianal abscesses in CGD occur due to the hampered influx or impaired function of granulocytes. Pseudomonas skin infection is common among PIDs especially patients with neutropenia [6,9]. PIDs characterized by distinctive susceptibility to Mycobacterium bovis in BCG (Bacille Calmette-Guérin) vaccine and serious complications ranging from prolonged local BCGitis and axillary lymphadenitis to disseminated disease (BCGosis) may occur in PIDs [10]. Since the routine national vaccination program in Libya includes BCG vaccine, PIDs, should be considered in any patient with prolonged BCGitis or BCGosis. High index of suspicion is important and needed for early diagnosis and intervention with anti-TB drugs. PIDs have increased susceptibility to fungal infections; yeasts, molds and dermatophyte; candida infection of the skin and mucous membranes may be the first sign of PIDs.

\begin{tabular}{|c|c|c|c|c|}
\hline PID & No. of Cases & Skin Infection (\%) & Eczema (\%) & Other Skin Manifestation (\%) \\
\hline \multicolumn{5}{|l|}{ Phagocytic } \\
\hline CGD & 34 & $\begin{array}{c}\text { Abscess }(75 \%) \\
\text { Perianal Fistulas (30\%) } \\
\text { Cellulitis }(9 \%) \\
\text { BCGitis }(20 \%) \\
\text { BCGosis }(15 \%) \\
\text { Tinea Corporis }(3 \%) \\
\text { Aspergillosis }(3 \%)\end{array}$ & - & $\begin{array}{l}\text { Granuloma (15\%) } \\
\text { Erythroderma (3\%) }\end{array}$ \\
\hline Neutropenia & 2 & $\begin{array}{l}\text { Abscess (100\%) } \\
\text { Ecthyma Gangrenosum (50\%) }\end{array}$ & - & - \\
\hline LAD & 1 & - & - & - \\
\hline \multicolumn{5}{|c|}{ Well-Defined Syndromes } \\
\hline HIES & 5 & $\begin{array}{c}\text { Abscess }(100 \%) \\
\text { Herpes Simplex }(40 \%) \\
\text { Candidiasis }(100 \%) \\
\text { Tinea corporis }(20 \%)\end{array}$ & $100 \%$ & Erythroderma (20\%) \\
\hline AT & 4 & $\begin{array}{l}\text { Impetigo (25\%) } \\
\text { Mucocutaneous Leishmaniasis }(25 \%)\end{array}$ & - & Telangiectasia $(100 \%)$ \\
\hline WAS & 2 & - & $100 \%$ & Purpura (100\%) \\
\hline DiGeorge & 2 & Extensive Chickenpox (50\%) & - & - \\
\hline \multicolumn{5}{|l|}{ Humoral } \\
\hline AG & 5 & Furunculosis $(60 \%)$ & - & - \\
\hline CVID & 1 & - & - & $\begin{array}{c}\text { Autoimmune (100\%): } \\
\text { Idiopathic Thrombocytopenic Purpura, Vitiligo, Urticaria }\end{array}$ \\
\hline THG & 1 & - & - & - \\
\hline \multicolumn{5}{|c|}{ Immune Dysregulation } \\
\hline CHS & 2 & - & - & Fair skin \& hair (100\%) \\
\hline \multicolumn{5}{|c|}{ Innate Immunity Defects } \\
\hline EDV & 1 & Multiple Wars $(100 \%)$ & - & - \\
\hline
\end{tabular}


It may present as an oral thrush, paronychia, dystrophic nails, granuloma formation or erythroderma [6].

Opportunistic fungi as Aspergillus species may cause serious skin problems especially in CGD; the involved skin may show erythemato-violaceous and sometimes purulent-to-necrotic nodular lesions $[6,11]$. In our study cutaneous spread of aspergillosis from chest wall was seen in a CGD patient. Children with PID are rarely exposed to common viral infections as viral wart and molluscum contagiosum in contrasts with their high frequency in patients with acquired immunodeficiency, AIDS in particular. Herpes simplex virus is among the most common viral skin infections encountered in T-cell deficient patients. The symptoms are often impressive and severe $[5,6]$. Recurrent herpes simplex infection and Kaposi varicelliform eruption were seen in 2 brothers with autosomal recessive HIES. Epidermodysplasia verruciformis has predisposition to papilloma virus infections. A general immunodeficiency is not present. These warts constitute a pre-malignant condition; one case was reported in this study [6].

Mucocutaneous Leishmaniasis was reported in an 11 years girl with AT, chronic prolonged course with nasal and lip destruction mandating surgical debridement and repeated courses of pentostam and amphotericin. Presence of immune deficiency was a poor prognostic sign with poor response to treatment [12].

Eczematous dermatitis is another nonspecific cutaneous alteration seen in many PIDs including HIES, WAS, AG, CVID, AT and CGD. It was apredominant feature in HIES, it was of early onset in infancy, atypical and different from eczema in normal individuals in that it is chronic, extensive and refractory to treatment with tendency to become 2ry infected and to flare up with systemic infections [13].

The presence of skin granuloma in immune deficient children has been described since 1970, these granuloma have regularly been seen in CGD, CVID and AT patients, and may be a presenting sign. Histopathology confirms the diagnosis. Infectious granuloma occurs due to persistent antigenic stimulus (chronic infections) whereas noninfectious granulomas occur because of lack of negative feedback by oxygen radicals on proinflammatory cytokines and it may resolve spontaneously. Erythroderma may be the presenting sign of immunodeficiency. It may be due to infection, drug, and dermatitis or remains unknown. In this study, 2 patients presented with erythroderma; HIES with generalized dermatitis and CGD with disseminated BCG disease.

Auto-immune diseases occur regularly in PIDs, it may be due to insufficiently cleared persistent antigens, and defects in apoptosis routes $[6,14]$. In our study vitiligo and thrombocytopenic purpura were seen in a CVID patient.

\section{Conclusion}

Most PIDs have cutaneous manifestations which may be the presenting signs and serve as important clues for diagnosis. Skin infections characterize many PIDs, but noninfectious dermatoses including eczema, granulomas, erythroderma and autoimmune conditions are also seen, their presentations and course are usually suggestive of the PIDs diagnosis.

Awareness of the associations between skin diseases and primary immune deficiency and the knowledge of PIDs cutaneous features may aide in the early detection and management of these serious or life-threatening immunological defects.

\section{References}

1. Subbarayan A, Colarusso G, Hughes SM, Gennery AR, Slatter M, et al. (2011) Clinical Features That Identify Children With Primary Immunodeficiency Diseases. Pediatrics 127: 810-816.

2. Richard SE, Ochs HD, Winkelstein JA (2004) Immunodeficiency disorders: General consideration. In: Stiehm EE, Ochs HD, Winkelstein JA, (eds.). Immunologic disorders in infants \& children. Saunders, Philadelphia. Pg no: 289-356.

3. [No authors listed] (1995) Primary immunodeficiency diseases. Report of a WHO scientific group Clin Exp Immunol 99: 1-24.

4. Notarangelo LD, Fischer A, Geha RS, Jean-Laurent Casanova, Chapel H, et al. (2009) Primary immunodeficiencies: The International Union of Immunological Societies (IUIS) Primary Immunodeficiencies (PID) Expert Committee. J Allergy Clin Immunol 124: 1161-1178.

5. Moin A, Farhoudi A, Moin M, Pourpak Z, Bazargan N (2006) Cutaneous Manifestations of Primary Immunodeficiency Diseases in Children. Iran J Allergy Asthma Immunol 5: 121-126.

6. Smitt JHS, Wulffraat NM, Kuijpers TW (2005) The skin in primary immunodeficiency disorders. Eur J Dermatol 15: 425-432.

7. Berron-Ruiz A, Berron-Perez R, Ruiz-Maldonaolo R (2000) Cutaneous markers of primary immunodeficiency diseases in children. Pediatr Dermatol 17: 91-96.

8. Tausk FA, Winkelstein JA (2001) Cutaneous Manifestations of Primary Immunodeficency Disease. In: Provost TT, Flynn JA (eds.). Cutaneous Medicine. BC Decker Inc., Hamilton, London, UK. Pg no: 263-272.

9. Baro M, Marin MA, Ruiz-Contreras J, De Miguel SF, Sánchez-Díaz I (2004) Pseudomonas aeruginosa sepsis and ecthyma gangrenosum as initial manifestations of primary immunodeficiency. Eur J Pediatr 163: 173-174.

10. Movahedi Z, Norouzi S, Mamishi S, Rezaei N (2011) BCGiosis as a presenting feature of a child with chronic granulomatous disease. Braz J Infect Dis 15: 83-86.

11. Galimberti R, Kowalczuk A, Hidalgo PI, Gonzalez Ramos M, Flores V (1998) Cutaneous aspergillosis: a report of six cases. Br J Dermatol 139: 522-521.

12. Van Griensven J, Carrillo E, López-Vélez R, Lynen L, Moreno J (2014) Leishmaniasis in imunosuppressed individuals. Clin Microbiol Infect 20: 286-299.

13. Szczawinska-Poplonyk A, Kycler Z, Pietrucha B, Heropolitanska-Pliszka E, Breborowicz A, et al. The hyperimmunoglobulin E syndrome - clinical manifestation diversity in primary immune deficiency. Orphanet J Rare Dis 6: 76.

14. Pruszkowski A, Bodemer C, Fraitag S, Teillac-Hmael D Amoric JC, et al. (2000) Neonatal and infantile erythrodermas: a retrospective study of 51 patients. Arch Dermatol 136: 875-880. 